\title{
Role of Semantic Paradigms for Optimization of Language Mapping in Clinical fMRI Studies
}

\author{
D. Zacà, S. Jarso, and J.J. Pillai
}

\begin{abstract}
BACKGROUND AND PURPOSE: The optimal paradigm choice for language mapping in clinical fMRI studies is challenging due to the variability in activation among different paradigms, the contribution to activation of cognitive processes other than language, and the difficulties in monitoring patient performance. In this study, we compared language localization and lateralization between 2 commonly used clinical language paradigms and 3 newly designed dual-choice semantic paradigms to define a streamlined and adequate language-mapping protocol.
\end{abstract}

MATERIALS AND METHODS: Twelve healthy volunteers performed 5 language paradigms: Silent Word Generation, Sentence Completion, Visual Antonym Pair, Auditory Antonym Pair, and Noun-Verb Association. Group analysis was performed to assess statistically significant differences in fMRI percentage signal change and lateralization index among these paradigms in 5 ROls: inferior frontal gyrus, superior frontal gyrus, middle frontal gyrus for expressive language activation, middle temporal gyrus, and superior temporal gyrus for receptive language activation.

RESULTS: In the expressive ROIs, Silent Word Generation was the most robust and best lateralizing paradigm (greater percentage signal change and lateralization index than semantic paradigms at $P<.01$ and $P<.05$ levels, respectively). In the receptive region of interest, Sentence Completion and Noun-Verb Association were the most robust activators (greater percentage signal change than other paradigms, $P<.01$ ). All except Auditory Antonym Pair were good lateralizing tasks (the lateralization index was significantly lower than other paradigms, $P<.05$ ).

CONCLUSIONS: The combination of Silent Word Generation and $\geq 1$ visual semantic paradigm, such as Sentence Completion and Noun-Verb Association, is adequate to determine language localization and lateralization; Noun-Verb Association has the additional advantage of objective monitoring of patient performance.

ABBREVIATIONS: BOLD = blood oxygen level-dependent; IFG = inferior frontal gyrus; $\mathrm{MFG}=$ middle frontal gyrus; $\mathrm{LI}=$ lateralization index; $\mathrm{MNI}=\mathrm{Montreal}$ Neurological Institute; MTG = middle temporal gyrus; PSC = percentage signal change; SFG = superior frontal gyrus; STG = superior temporal gyrus

T he -fold aim of presurgical language mapping is to localize eloquent language cortical tissue and determine hemispheric language lateralization for surgical planning. To date, presurgical

Received November 21, 2012; accepted after revision January 26, 2013.

From the Division of Neuroradiology (D.Z., J.J.P.), Russell H. Morgan Department of Radiology and Radiological Science, Johns Hopkins University School of Medicine, Baltimore, Maryland; Center for Mind/Brain Sciences (D.Z.), University of Trento, Trento, Italy; and F.M. Kirby Research Center for Functional Brain Imaging (S.J.), The Kennedy-Krieger Institute, Baltimore, Maryland.

This work was supported in part by a research grant from Siemens Medical Solutions (Dr Pillai is the Principal Investigator). Siemens was not involved in the study design, data collection, analysis, or manuscript preparation but did provide salary support for Dr Zacà.

Paper previously presented in part at: 50 th Annual Meeting of the American Society of Neuroradiology, April 21-26, 2012; New York, New York.

Please address correspondence to Jay J. Pillai, MD, Division of Neuroradiology, Russell H. Morgan Department of Radiology and Radiological Science, The Johns Hopkins Hospital, Phipps B-100, 1800 Orleans St, Baltimore, MD 21287; e-mail: jpillail@jhmi.edu

http://dx.doi.org/10.3174/ajnr.A3628 language mapping is challenged by the inability to a priori define an individual's language network. ${ }^{1}$ The classically accepted model of language representation, describing expressive language (speech production) in the left inferior frontal gyrus (IFG) (Broca area) and language comprehension (receptive language processing) in the Wernicke area, in the posterior aspect of the left superior temporal gyrus (STG), is inadequate to fully describe the entire language network. A newer framework, validated by both functional and structural MR imaging, proposes that language processing occurs through a ventral and dorsal pathway. ${ }^{2}$

Other limitations of clinical language blood oxygen level-dependent (BOLD) fMRI include both variability in activation patterns among different language paradigms and the variable specificity of activation for the delineation of the essential language cortex. Cognitive processes such as decision-making, attention, and working memory are also involved in language processing, thereby making it more difficult to explicitly use language mapping to distinguish essential and nonessential regions of the language network. 


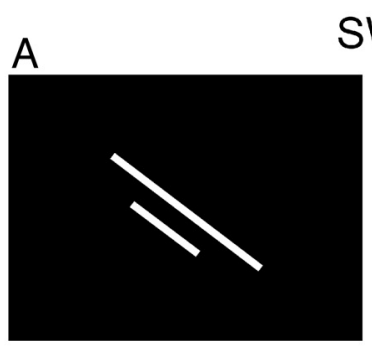

SWG

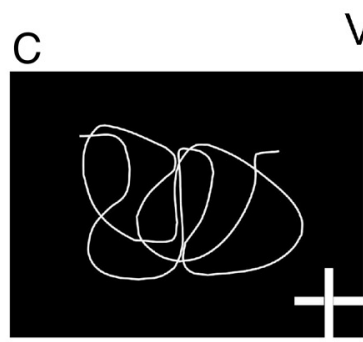

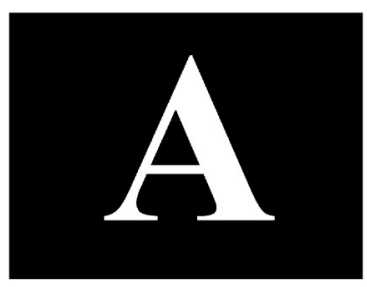

VAP

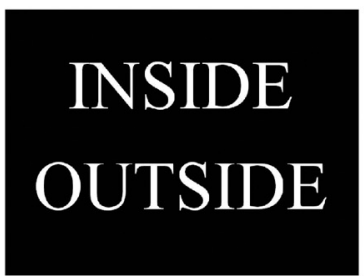

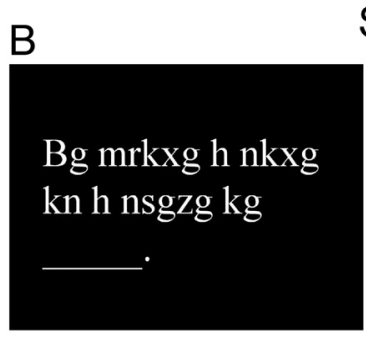

SC

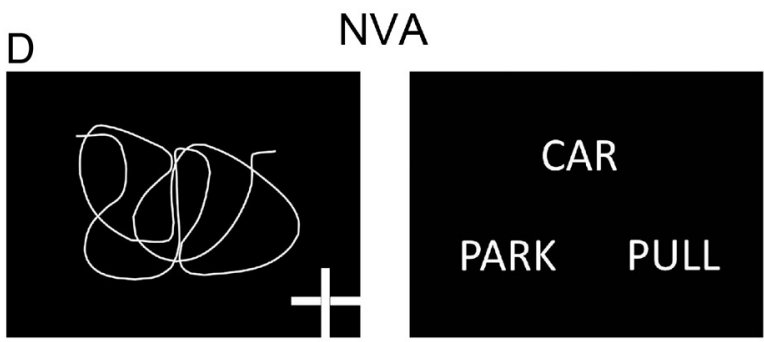

FIG 1. Examples of the stimuli in the control (first and third columns) and active (second and fourth columns) tasks in the 4 visually delivered paradigms used in the study: A, Silent Word Generation; $B$, Sentence Completion; $C$, Visual Antonym Pair; $D$, Noun-Verb Association.

Additionally, monitoring patient compliance during task performance is important to assess the task-activation pattern. Covert verbal fluency tasks have been shown to be the best for determination of expressive language regions and hemispheric lateralization, but they do not allow adequate monitoring of task performance inside the scanner. ${ }^{3}$

Determination of language lateralization is, in many cases, as important as language localization for surgical planning. The risk of postoperative deficits is generally thought to be higher in surgical procedures in which the lesion is located in the language-dominant hemisphere. However, the assessment of language lateralization by fMRI can be affected by many factors, including task selection, ROIs used for lateralization index (LI) computation, and statistical thresholding. 4,5

For all of the above-mentioned reasons, fMRI for language mapping is still not universally accepted as a standard of care for presurgical planning. In light of the recently developed language models and to contribute to the effort to establish effective protocols for language $\mathrm{fMRI}$, we designed this study to determine which paradigm or combination of paradigms provides an adequate and streamlined protocol for comprehensive language mapping in clinical fMRI studies. This was carried out by comparing patterns of activation between 2 commonly used clinical language fMRI paradigms and 3 newly designed semantic language tasks that allow objective monitoring of patient task performance.

\section{MATERIALS AND METHODS \\ Study Design}

Twelve right-handed ( 6 men/ 6 women; range, $21-45$ years of age) primarily English-speaking healthy volunteers participated in this study approved by the institutional review board. Two commonly used clinical language paradigms-Silent Word Generation and Sentence Completion-and 3 newly designed semantic paradigms_-Visual Antonym Pair, Auditory Antonym Pair, and Noun-Verb Association-were performed by all the participating subjects (Fig 1). All paradigms were block design with alternating active and control blocks lasting 20 seconds each for a total of 4 minutes. The paradigms were implemented by using Prism Acquire (Prism Clinical Imaging Inc, Elm Grove, Wisconsin). See below for a description of each paradigm:

\section{Silent Word Generation}

Control Block. Visual fixation on 2 consecutive nonsense drawings, each for 10 seconds.

Active Block. Covert generation of words for 2 consecutively presented letters, each for 10 seconds.

\section{Sentence Completion}

Control Block. Scan through 5 consecutive samples of scrambled letters arranged to resemble words in a sentence.

Active Block. Covert reading of 5 consecutive real sentences with the last word missing and covert generation of a word to complete each sentence.

\section{Visual Antonym Pair}

Control Block. Visual fixation of 5 consecutive drawings with a cross placed in 1 of the 4 corners of the screen. Keypad button press was required if the cross location was in the upper or lower right corner.

Active Block. Reading of 5 consecutive pairs of words. Keypad button press was required if the 2 words were antonyms.

\section{Auditory Antonym Pair}

Control Block. Listening to 5 consecutive pairs of tones. Keypad button press was required if the 2 tones were identical.

Active Block. Listening to 5 consecutive pairs of words. Keypad button press was required if the 2 words were antonyms.

\section{Noun-Verb Association}

Control Block. Same as "Visual Antonym Pair."

Active Block. Visual presentation of 5 samples of a noun on the top row and a pair of verbs on the bottom row. Keypad button 

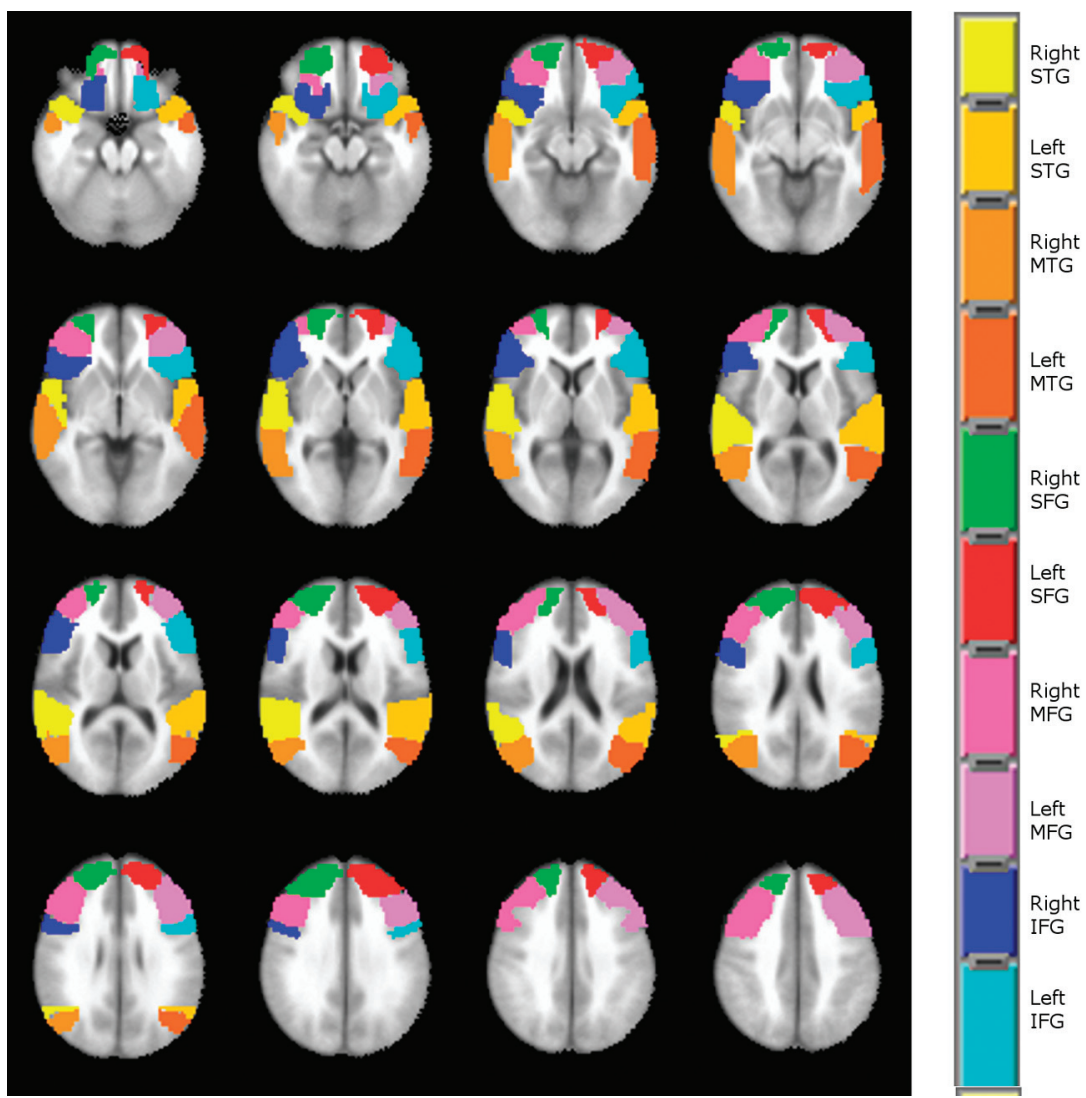

FIG 2. Regions of interest defined on the MNI-152 atlas, where language localization (PSC) and lateralization (LI) were compared among the 5 language paradigms adopted in this study. dient-echo sequence $(\mathrm{TR}=2300 \mathrm{~ms}$; $\mathrm{TI}=900 \mathrm{~ms} ; \mathrm{TE}=3.5 \mathrm{~ms}$; flip angle $=$ $9^{\circ} ; \mathrm{FOV}=256 \mathrm{~cm}^{2} ; 256 \times 256$ matrix acquisition; section thickness $=1 \mathrm{~mm}$ ).

\section{Image Analysis}

AFNI software (http://afni.nimh.nih. gov/afni) was used for image processing. Preprocessing included section timing, motion correction, spatial smoothing, and registration in a stereotactic space, the Montreal Neurological Institute (MNI)152 atlas. Regression analysis was then performed by fitting each voxel time-series, divided by the mean and multiplied by 100 , to a theoretic expected time-series (ideal TS) generated by convoluting each paradigm timing with a hemodynamic impulse response function. Percentage signal change (PSC) maps from the baseline were calculated for each paradigm as

$$
P S C=100 \times \frac{a \times P P(\text { ideal TS })}{\text { Baseline }}
$$

where PP indicates peak to peak; PP (ideal TS), maximum (ideal TS) to minimum (ideal TS); and Baseline $=\mathrm{b}_{0}+$ $\mathrm{b}_{1} \times$ (average polynomial grade 1$)+$ $a \times$ minimum (ideal TS), where $\mathrm{b}_{0}$ is the press was required if the verb presented on the right of the bottom row was more closely semantically associated with the presented noun than the verb on the bottom left (eg, Fig 1D; the verb "park" on the bottom left was more closely associated with the noun "car" than the verb "pull " located on the bottom right; therefore, a button response was not required in this case; vice versa, if the verb "park" was on the bottom right and the verb "pull," on the bottom left, a button response would have been required).

For the dual-choice tasks, the number of expected button presses in the control and active blocks was balanced. A training and practice session was performed outside the MR imaging scanner with each participant to provide task instructions and opportunity to practice the tasks by using similar but different stimuli from those that were included in the actual examination.

\section{Imaging}

Images were acquired by using a 3T Magnetom Trio scanner (Siemens, Erlangen, Germany) equipped with a 12-channel head matrix coil.

BOLD images were acquired by using a single-shot $\mathrm{T} 2^{\star} \mathrm{WI}$ gradient-echo EPI sequence. Imaging parameters were the following: $\mathrm{TR}=2000 \mathrm{~ms}$; $\mathrm{TE}=30 \mathrm{~ms}$; flip angle $=90^{\circ} ; \mathrm{FOV}=24 \mathrm{~cm}$; $80 \times 80$ matrix acquisition; section thickness $=3 \mathrm{~mm}$ with a 1-mm gap between sections.

Structural images for coregistration and overlay of functional activation maps were acquired by using a standard 3D T1WI gra- baseline constant, $b_{1}$ a linear trend slope, and $a$ the regression coefficient.

\section{Statistical Analysis}

Language localization and lateralization were compared among the 5 paradigms in 5 ROIs (IFG, superior frontal gyrus [SFG], middle frontal gyrus [MFG], middle temporal gyrus [MTG] and STG) automatically defined for the left and right hemispheres on the MNI atlas available in AFNI (Fig 2). To assess statistically significant differences in language localization, we performed a nonparametric analysis with statistical significance considered at the $P<.01$ level. A Friedman test was run voxelwise in each region of interest followed by Wilcoxon signed rank tests between each pair of paradigms in the voxels where the Friedman test score achieved statistical significance. Multiple comparison correction was applied on the results of the Wilcoxon tests by using a combination of probability and clustering thresholding obtained by using the AlphaSim simulation program available in AFNI.

Lateralization was expressed by calculating, in each region of interest for each paradigm, the LI by using a threshold-independent method $^{6}$ :

$$
L I=\frac{L H-R H}{L H+R H}
$$

where $\mathrm{LH}$ and $\mathrm{RH}$ are the weighted sum of all voxel $t$ values in the left and right hemisphere portion, respectively, of each region of interest. 
Table 1: Summary of Wilcoxon test results in PSC for language localization in the 5 ROIs included in the analysis

\begin{tabular}{|c|c|c|c|c|}
\hline IFG & MFG & SFG & MTG & STG \\
\hline AAP $<$ NVA & $\mathrm{AAP}<\mathrm{NVA}$ & $\mathrm{AAP}<\mathrm{NVA}$ & $A A P>N V A$ & $A A P>N V A$ \\
\hline Left & Left & Left & Right & Right \\
\hline $\mathrm{SC}>\mathrm{AAP}$ & $\mathrm{AAP}>\mathrm{NVA}$ & $A A P>N V A$ & $\mathrm{SC}>\mathrm{AAP}$ & $S C>N V A$ \\
\hline Left & Right & Right & Left & Right \\
\hline$S C>N V A$ & $\mathrm{SC}>\mathrm{AAP}$ & $\mathrm{SC}>\mathrm{AAP}$ & $\mathrm{SC}>\mathrm{VAP}$ & $\mathrm{SC}>\mathrm{VAP}$ \\
\hline Left & Left & Left & Right and Left & Right \\
\hline$S C>V A P$ & $S C>N V A$ & $\mathrm{SC}>\mathrm{VAP}$ & SWG $<$ AAP & $\mathrm{SWG}<\mathrm{AAP}$ \\
\hline Left & Left & Left & Right and Left & Right and Left \\
\hline SWG $>$ AAP & $\mathrm{SC}>\mathrm{VAP}$ & SWG $>$ AAP & SWG $<$ NVA & SWG $<$ NVA \\
\hline Left & Left & Left & Right and Left & Right and Left \\
\hline SWG $>$ NVA & $S W G>A A P$ & SWG $>$ NVA & $\mathrm{SWG}<\mathrm{SC}$ & $\mathrm{SWG}<\mathrm{SC}$ \\
\hline Left & Left & Left & Right and Left & Right and Left \\
\hline SWG $>$ VAP & SWG $>$ VAP & SWG $>$ VAP & $\mathrm{SWG}<\mathrm{VAP}$ & $\mathrm{SWG}<\mathrm{VAP}$ \\
\hline Left & Left & Left & Right and Left & Right and Left \\
\hline VAP $>$ AAP & $\mathrm{SWG}<\mathrm{VAP}$ & $\mathrm{VAP}>\mathrm{AAP}$ & $\mathrm{VAP}<\mathrm{AAP}$ & $\mathrm{VAP}<\mathrm{AAP}$ \\
\hline Left & Right & Left & Right & Right and Left \\
\hline $\mathrm{SC}>\mathrm{AAP}$ & VAP $>$ AAP & $\mathrm{VAP}<\mathrm{AAP}$ & $S C>N V A$ & \\
\hline Left & Left & Right & Right & \\
\hline
\end{tabular}

Note:-AAP indicates Auditory Antonym Pair; NVA, Noun-Verb Association; SC, Sentence Completion; SWG, Silent Word Generation; VAP, Visual Antonym Pair.

Table 2: Summary of Wilcoxon test results in the LI for lateralization analysis

\begin{tabular}{llll}
\hline \multicolumn{1}{c}{ IFG } & \multicolumn{1}{c}{ MFG } & \multicolumn{1}{c}{ SFG } & \multicolumn{1}{c}{ MTG } \\
\hline SWG $>$ SC & SWG $>$ SC & SWG $>$ AAP & SWG $>$ AAP \\
SWG $>$ AAP & SWG $>$ VAP & SWG $>$ NVA & SC $>$ AAP \\
NVA $>$ AAP & SWG $>$ NVA & SC $>$ NVA & VAP $>$ AAP \\
NVA $>$ VAP & SWG $>$ AAP & & NVA $>$ AAP \\
& NVA $>$ AAP (trend) & & \\
\hline
\end{tabular}

Note:-AAP indicates Auditory Antonym Pair; NVA, Noun-Verb Association; SC, Sentence Completion; SWG, Silent Word Generation; VAP, Visual Antonym Pair.

The same nonparametric tests used for language localization analysis were performed to assess differences in LI among the paradigms in the 5 ROIs. Statistical significance was considered at the $P<.05$ level.

\section{RESULTS}

\section{Language Localization}

All 5 ROIs showed voxels with $\chi$ scores above the threshold associated with statistical significance $(P<.01)$. Table 1 summarizes the significant results of the Wilcoxon tests comparing the PSC between pairs of paradigms in each region of interest. The results of the simulation run in AlphaSim determined a cluster size of 9 voxels to apply multiple comparison correction on the Wilcoxon test $\chi$ score maps at the $P<.01$ level. The verbal fluency paradigm Silent Word Generation and Sentence Completion were demonstrated to be more robust activators than the semantic paradigms in frontal gyri ROIs (IFG, MFG, SFG) in the dominant left hemisphere, with Auditory Antonym Pair showing the weakest activation in the left IFG, MFG, and SFG but stronger than Visual Antonym Pair and Noun-Verb Association in the right IFG, MFG, and SFG. Silent Word Generation provided the weakest activation in both the right and left MTG and STG. Sentence Completion was more robust than Auditory Antonym Pair and Visual Antonym Pair in the left MTG and STG, but not more robust than Noun-Verb Association. Auditory Antonym Pair and Sentence Completion were the strongest activators of the right MTG and STG.

\section{Language Lateralization}

In Table 2, the results of the Wilcoxon tests demonstrating a significant difference at $P<.05$ in LI between each pair of paradigms in each region of interest are reported. Silent Word Generation was the best lateralizing task in the expressive ROIs, whereas Sentence Completion did not outperform, in general, Noun-Verb Association and Visual Antonym Pair as a lateralizing task, except for Noun-Verb Association in the SFG. Auditory Antonym Pair provided the most bilateral activation both in the frontal regions and in the MTG. The other 4 paradigms did not demonstrate statistically significant differences in LI in the MTG. No significant differences in LI between the paradigms were found in the STG.

\section{DISCUSSION}

In this study, we compared the localization (as locally detectable statistically significant PSC) and lateralization (via the LI) provided by 2 clinically used covert language tasks, Silent Word Generation and Sentence Completion, with those provided by 3 newly designed dual-choice block-design semantic language paradigms, Visual Antonym Pair, Auditory Antonym Pair, and Noun-Verb Association in a group of right-handed healthy volunteers. We made this comparison to determine which paradigm or set of paradigms would be most useful for presurgical mapping of the eloquent language cortex. The analyzed ROIs included more than just the classic Broca and Wernicke areas (left IFG and left STG, respectively), because it is well-recognized that language activation extends well beyond these 2 areas. ${ }^{2}$ Language is not a unitary process but rather a collection of processes operating at distinct levels, such as phonetics, phonology, orthography, and semantics. Therefore, the representation areas related to these processes deserve attention in presurgical planning because their inadvertent resection may also cause postoperative language deficits. We adopted a nonparametric statistical approach because the variables that we compared among the different tasks in the different ROIs for localization (PSC) and lateralization (LI) are defined as ratios of Gaussian variables, and in general, the distribution of a quotient of 2 normal variables can be multimodal. 
The results demonstrate that Silent Word Generation is the most robust task for language localization and the most effective for determining language lateralization in the frontal gyri (IFG, MFG, and SFG) of the dominant (left) language hemisphere (Tables 1 and 2). A verbal fluency task, such as Silent Word Generation, requires phonologic access, verbal working memory, and lexical search activity, and these functions are localized in the left inferior frontal gyrus as demonstrated in multiple studies. ${ }^{7,8}$ In addition, the MFG and SFG are regions of the brain involved in speech production because of the activation of the middle frontal cortex in word retrieval ${ }^{9}$ and the presupplementary motor area for initiation and execution. ${ }^{10}$ Sentence Completion activates the dominant hemisphere frontal gyri as robustly as Silent Word Generation because of the word-generation component present in this paradigm (a word required to complete each sentence). However, its pattern of activation is significantly less lateralized than Silent Word Generation in the IFG and MFG because of the contribution of the right hemisphere homologous areas in speech comprehension tasks involving executive processing. ${ }^{11}$ Semantic decision tasks also elicit activation in the IFG, MFG, and SFG, ${ }^{12}$ but the lack of word retrieval, initiation, and execution components of the Noun-Verb Association, Visual Antonym Pair, and Auditory Antonym Pair tasks explains their weaker activation in these gyri compared with our 2 tasks with a verbal fluency component. In addition, the auditory semantic task Auditory Antonym Pair showed weaker activation than Visual Antonym Pair and Noun-Verb Association. This result is consistent with the findings of multiple studies reporting activation of the pars triangularis and pars opercularis both for speech and nonspeech sounds when they had to be held in auditory working memory, as in both the control and active blocks of the Auditory Antonym Pair task. ${ }^{13,14}$

The requirement of working memory and articulary recording activity both in the control and active tasks for Auditory Antonym Pair justifies the same findings in the MFG and SFG. ${ }^{15,16}$ The analysis of patterns of activation in the temporal gyri demonstrated a weaker BOLD PSC for the purely verbal fluency task Silent Word Generation both in the left and right hemispheres compared with the other 4 paradigms, all of which are associated with semantic processing localized in the middle temporal and angular gyri. ${ }^{17}$ The relative strength of activation among Sentence Completion, Noun-Verb Association, Visual Antonym Pair, and Auditory Antonym Pair was somewhat variable depending also on the considered hemisphere. In particular, as reported in Table 1, Auditory Antonym Pair was the most robust activator task in the right MTG and STG because its PSC in these 2 regions of interest was greater than Silent Word Generation, Noun-Verb Association, and Visual Antonym Pair in both regions. Auditory Antonym Pair activation results are also highly bilateral in the STG (average LI $=0.20 \pm 0.20$ ) and in the MTG (average LI $=$ $0.15 \pm 0.20)$, where it was also significantly lower in comparison with the other 4 paradigms $(P<.05)$.

Results from the literature indicate a bilateral superior temporal activation for both speech and nonspeech sounds. ${ }^{18}$ However, the reason for bilateral STG activation even after the subtraction of the speech (tones) control task from the speech (antonyms) active task may be that more demand is placed on short-term auditory memory during the speech task than during the tone task. Furthermore, contrary to phonologic processing studies reporting lateralized activation in receptive language areas, the semantic component of the active task (antonym versus nonantonym) explains the bilateral pattern of activation in our group of volunteers. ${ }^{4}$ We found, in our study, greater activation for Sentence Completion in the left MTG compared with Visual Antonym Pair and Auditory Antonym Pair but not compared with Noun-Verb Association, and these findings could be attributed to the higher level of lexical-semantic processing required for Sentence Completion and Noun-Verb Association. ${ }^{19}$ Instead Sentence Completion activation was stronger than Noun-Verb Association and Visual Antonym Pair in the right MTG and STG, confirming the role of the right hemisphere in semantic tasks involving executive processing. ${ }^{11}$

The Silent Word Generation group LI was not significantly different from either Sentence Completion or the semantic paradigms but was higher than that of Auditory Antonym Pair. Therefore one could consider using only Silent Word Generation in a clinical language fMRI examination because of its capability to determine language lateralization in both the frontal and temporal lobes. However its PSC is weaker in both the left and right temporal gyri compared with the other 4 paradigms that include language comprehension in the active blocks of the paradigms; therefore, the semantic tasks are more adequate to map the temporal regions involved in the language network because they elicit a stronger BOLD response than a verbal fluency task. Furthermore, it has been demonstrated that the combination of multiple tasks, a language-specific region of interest approach and implementation of statistical threshold-independent approaches for determination of hemispheric lateralization, provides more reliable lateralization that correlates better with the criterion standard Wada test. ${ }^{20,21}$

One limitation of this study includes the exclusion of the cerebellum from region-of-interest analysis. The cerebellum has demonstrated fMRI activation during silent articulation. ${ }^{22}$ Cases of cognitive deficits in association with cerebellar damage have been reported, ${ }^{23}$ yet frank aphasic disturbances are rare. Multiple cognitive studies performed on a group of healthy volunteers have demonstrated a further parceling out of language functions in each of the 5 ROIs considered in our work. ${ }^{24,25}$ However, since this study aimed to improve language presurgical mapping, where analysis is conducted at the single subject level, such parcellation would not be practical and it most likely would not add any critical information for patient surgical management. Finally, no Wada testing was performed to actually confirm language lateralization in these subjects, but this would not be ethical in a group of healthy volunteers.

\section{CONCLUSIONS}

In this study, we demonstrated that the combination of a fluency task such as Silent Word Generation and $\geq 1$ visually delivered semantic task provides an adequate protocol for determination of language localization and lateralization. The newly designed Noun-Verb Association paradigm is potentially very effective because its forced dual-choice paradigm design allows effective patient monitoring of task performance. Nevertheless, it is always 
advisable in clinical studies to have a variety of available paradigms because if a patient finds a particular task too challenging because of neurologic impairment, it will rarely produce meaningful fMRI results.

\section{ACKNOWLEDGMENTS}

The authors thank Gayane Yenokyan, $\mathrm{MD}, \mathrm{PhD}$, for assistance with data analysis.

Disclosures: Domenico Zacà—RELATED: Grant: Siemens Medical Solutions, * Comments: Siemens provided support for my postdoctoral fellowship salary at Johns Hopkins University. Jay J. Pillai-RELATED: Grant: Siemens Medical Solutions, ${ }^{*}$ Comments: This research grant paid for Dr Zacà's (my former postdoctoral fellow) salary. However, Siemens was not involved in the study design, analysis, reporting of results, or writing of the manuscript in any way, OTHER RELATIONSHIPS: I am an unpaid member of the Medical Advisory Board of Prism Clinical Imaging. *Money paid to the institution.

\section{REFERENCES}

1. Seghier ML, Lazeyras F, Pegna AJ, et al. Variability of fMRI activation during a phonological and semantic language task in healthy subjects. Hum Brain Mapp 2004;23:140-55

2. Saur D, Kreher BW, Schnell S, et al. Ventral and dorsal pathways for language. Proc Natl Acad Sci U S A 2008;105:18035-40

3. Partovi S, Konrad F, Karimi S, et al. Effects of covert and overt paradigms in clinical language fMRI. Acad Radiol 2012;19:518-25

4. Zacà D, Nickerson JP, Deib G, et al. Effectiveness of four different clinical fMRI paradigms for preoperative regional determination of language lateralization in patients with brain tumors. Neuroradiology 2012;54:1015-25

5. Ruff IM, Petrovich Brennan NM, Peck KK, et al. Assessment of the language laterality index in patients with brain tumor using functional MR imaging: effects of thresholding, task selection, and prior surgery. AJNR Am J Neuroradiol 2008;29:528-35

6. Branco DM, Suarez RO, Whalen S, et al. Functional MRI of memory in the hippocampus: laterality indices may be more meaningful if calculated from whole voxel distributions. Neuroimage 2006;32:592-602

7. Palmer ED, Rosen HJ, Ojemann JG, et al. An event-related fMRI study of overt and covert word stem completion. Neuroimage 2001; 14(1 pt 1):182-93

8. Eulitz C, Elbert T, Bartenstein P, et al. Comparison of magnetic and metabolic brain activity during a verb generation task. Neuroreport 1994;6:97-100

9. Paulesu E, Goldacre B, Scifo P, et al. Functional heterogeneity of left inferior frontal cortex as revealed by fMRI. Neuroreport 1997;8:2011-17
10. Kawashima R, Okuda J, Umetsu A, et al. Human cerebellum plays an important role in memory-timed finger movement: an fMRI study. J Neurophysiol 2000;83:1079-87

11. Vigneau M, Beaucousin V, Herve PY, et al. What is right-hemisphere contribution to phonological, lexico-semantic, and sentence processing? Insights from a meta-analysis. Neuroimage 2011;54:577-93

12. Binder JR, Desai RH, Graves WW, et al. Where is the semantic system? A critical review and meta-analysis of 120 functional neuroimaging studies. Cereb Cortex 2009;19:2767-96

13. Burton MW, Small SL, Blumstein SE. The role of segmentation in phonological processing: an fMRI investigation. J Cogn Neurosci 2000;12:679-90

14. Hsieh L, Gandour J, Wong D, et al. Functional heterogeneity of inferior frontal gyrus is shaped by linguistic experience. Brain Lang 2001;76:227-52

15. Wilson SM, Iacoboni M. Neural responses to non-native phonemes varying in producibility: evidence for the sensorimotor nature of speech perception. Neuroimage 2006;33:316-25

16. Wilson SM, Saygin AP, Sereno MI, et al. Listening to speech activates motor areas involved in speech production. Nat Neurosci 2004;7:701-02

17. Vandenberghe $\mathrm{R}$, Price $\mathrm{C}$, Wise $\mathrm{R}$, et al. Functional anatomy of a common semantic system for words and pictures. Nature 1996;383:254-56

18. Booth JR, Burman DD, Meyer JR, et al. Functional anatomy of intraand cross-modal lexical tasks. Neuroimage 2002;16:7-22

19. Démonet JF, Chollet F, Ramsay S, et al. The anatomy of phonological and semantic processing in normal subjects. Brain 1992;115(pt 6): $1753-68$

20. Niskanen E, Kononen M, Villberg V, et al. The effect of fMRI task combinations on determining the hemispheric dominance of language functions. Neuroradiology 2012;54:393-405

21. Suarez RO, Whalen S, Nelson AP, et al. Threshold-independent functional MRI determination of language dominance: a validation study against clinical gold standards. Epilepsy Behav 2009;16:288-97

22. Ackermann H. Cerebellar contributions to speech production and speech perception: psycholinguistic and neurobiological perspectives. Trends Neurosci 2008;31:265-72

23. Fiez JA, Petersen SE, Cheney MK, et al. Impaired non-motor learning and error detection associated with cerebellar damage: a single case study. Brain 1992;115(pt 1):155-78

24. Specht K, Reul J. Functional segregation of the temporal lobes into highly differentiated subsystems for auditory perception: an auditory rapid event-related fMRI-task. Neuroimage 2003;20:1944-54

25. Thierry G, Giraud AL, Price C. Hemispheric dissociation in access to the human semantic system. Neuron 2003;38:499-506 